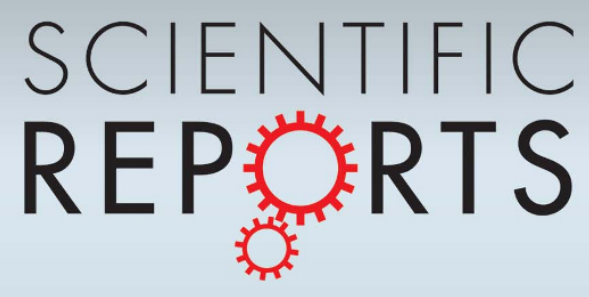

OPEN

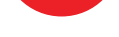

SUBJECT AREAS: BIOPHYSICAL CHEMISTRY

CHEMICAL BIOLOGY

ENZYMES

PROTEIN DESIGN

Received

20 January 2011

Accepted

21 March 2011

Published

14 June 2011

Correspondence and requests for materials should be addressed to

O.M. (omillet@

cicbiogune.es)

\title{
Halophilic enzyme activation induced by salts
}

\author{
Gabriel Ortega, Ana Laín, Xavier Tadeo, Blanca López-Méndez, David Castaño \& Oscar Millet
}

Contribution from the Structural Biology Unit, CIC bioGUNE, Bizkaia Technology Park, Building 800, 48160 Derio, Spain.

Halophilic archea (halobacteriae) thrive in hypersaline environments, avoiding osmotic shock by increasing the ion concentration of their cytoplasm by up to 3-6 M. To remain folded and active, their constitutive proteins have evolved towards a biased amino acid composition. High salt concentration affects catalytic activity in an enzyme-dependent way and a unified molecular mechanism remains elusive. Here, we have investigated a DNA ligase from Haloferax volcanii $(\mathrm{Hv} \mathrm{LigN})$ to show that $\mathrm{K}^{+}$triggers catalytic activity by preferentially stabilising a specific conformation in the reaction coordinate. Sodium ions, in turn, do not populate such isoform and the enzyme remains inactive in the presence of this co-solute. Our results show that the halophilic amino acid signature enhances the enzyme's thermodynamic stability, with an indirect effect on its catalytic activity. This model has been successfully applied to reengineer $\mathrm{HV}$ LigN into an enzyme that is catalytically active in the presence of $\mathrm{NaCl}$.

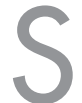

alines are harsh environments where the high salt concentration precludes the survival of almost any living form but the halophilic archaea. To avoid osmotic shock, these extremophiles equilibrate their ionic strength with the environment, and $\mathrm{K}^{+}\left(\right.$or $\mathrm{Na}^{+}$) ions can increase the cytoplasm's osmolarity up to 4 Osm/L ${ }^{1}$. To remain folded and active at this high intracellular ionic concentration, proteins from halophilic organisms have evolved towards a biased amino acid composition: glutamate and aspartate are found at higher frequencies compared to their mesophilic counterparts ${ }^{2-4}$. This increase in acidic residues is accompanied by a low content of bulky hydrophobic side chains and a drastic reduction in the number of lysines. Such amino acid composition places halophilic proteins amongst the most acidic proteins naturally found ${ }^{5}$. Genomic analyses of taxonomically unrelated species reflect similar signatures in the amino acid composition, indicating that this is a universal evolutionary trait ${ }^{2}$.

Biophysical characterization of a plethora of examples has demonstrated that salt $(\mathrm{NaCl}$ and/or $\mathrm{KCl}$ ) always increases the stability of halophilic proteins ${ }^{6,7}$. More detailed studies in which proper thermodynamic descriptors were obtained also support this conclusion ${ }^{8,9}$. The monotonic stabilization of halophilic proteins by salt contrasts with the complex effect that $\mathrm{NaCl}$ or $\mathrm{KCl}$ has on the functionality of halophilic enzymes ${ }^{10,11}$ : some of them decrease their catalytic activity at increasing salt concentrations, showing suboptimal activity at physiological ionic strength ${ }^{12,13}$. On the other hand, proteins like $\beta$-galactosidase from Haloferax alicantei are activated by salt ${ }^{14}$ and, perhaps more strikingly, the two activities for the catalase peroxidase from Halobacterium salinarium show contrasting dependence on $\mathrm{NaCl}$ concentration ${ }^{15}$. Some reports indicate that the activity of halophilic enzymes can also be modulated by anions other than chloride ${ }^{7,16}$, but a unified molecular mechanism to explain these differences remains elusive.

Ligase $\mathrm{N}$ from Haloferax volcanii $(\mathrm{H} v \operatorname{LigN})$ is a $\mathrm{NAD}^{+}$-dependent enzyme that joins the end of DNA molecules during replication and repair ${ }^{17}$. Full length $H v$ LigN has the characteristic amino acid composition found in halophilic proteins (Figure 1A) ${ }^{2,8}$. Such features allow the protein to remain folded in hypersaline environments and $H v$ LigN requires $\mathrm{KCl}$ to be active and shows increased activity upon addition of this salt. Surprisingly, the enzyme remains inactive in the presence of $\mathrm{NaCl}^{17}$. The full length LigN has 699 amino acids and shares its domain structure with all the bacterial $\mathrm{NAD}^{+}$-dependent ligases including a DNA binding domain, an adenylation domain (divided into the sub-domains $1 \mathrm{~A}$ and $1 \mathrm{~B}$ ) and an $\mathrm{OB}$ fold domain ${ }^{17}$ (Figure 1B). During catalysis, an AMP molecule is transferred from $\mathrm{NAD}^{+}$to the protein (adenylation of ligase) resulting in the closure of subdomains $1 \mathrm{~A}$ and $1 \mathrm{~B}^{18}$. Here, we have investigated the putative coupling between changes in stability induced by salt and its effect on enzyme activity. Our results show that, unlike $\mathrm{Na}^{+}$, potassium ion has the ability to stabilize the enzyme as well as to modulate $H v$ LigN ligase activity. The two mechanisms are coupled, and the differential effect of salt on two different enzyme conformations, both part of the reaction pathway, is the key element to understanding the activation of the enzyme by $\mathrm{KCl}$, the lack of activation by $\mathrm{NaCl}$ and the effect induced by other salts. 


\section{Results}

Effect of $\mathrm{K}^{+}$on the enzymatic activity and the thermodynamic stability of $\mathrm{Hv}$ LigN. The ligase activity of $\mathrm{Hv}$ LigN is fully dependent on the $\mathrm{KCl}$ concentration: the enzyme cannot ligate DNA in the absence of this salt, it increases its activity upon $\mathrm{KCl}$ addition and it reaches a measurable maximum at $3.2 \mathrm{M} \mathrm{KCl}^{19} . \mathrm{Hv}$ LigN selectively joins fragments I (8454 bp) and IV (5686 bp) of $B s t$ EII-digested $\lambda$ DNA to produce a new oligomer that runs in an agarose gel at the molecular weight of the sum of the two fragments ${ }^{19}$. This reaction was used to show that $H v \operatorname{LigN}$ activity is dependent on $\mathrm{KCl}$ concentration up to $3.2 \mathrm{M}$ (Figures $1 \mathrm{C}$ and $\mathrm{S} 1$ ).

It has been shown in the literature that the $\mathrm{NAD}^{+}$-dependent ligase $\mathrm{N}$ from Enterococcus faecalis undergoes a conformational rearrangement upon adenylation, resulting in the closure of subdomains $1 \mathrm{~A}$ and $1 \mathrm{~B}^{20}$. Using homology programs ${ }^{21,22}$ and the available high resolution structures ${ }^{18}$, we modelled the deadenylated ( $\mathrm{Hv}$ deaLigN, open) and adenylated ( $H v$ ade-LigN, closed) conformations of the adenylation domain in $H v$ LigN (Figure 1B). The modelled structures retained the fold of the mesophilic counterparts, consistent with the fact that halophilic proteins concentrate the changes in the surface ${ }^{23,24}$. Interestingly, the adenylated protein has less exposed area, burying $331 \AA^{2}$ more than $H v$ dea-LigN to the solvent. Adenylation of LigN is a required step for DNA ligation to occur, and the two conformations correspond to two instances in the enzymatic reaction coordinate.

In contrast to thermal denaturation, $H v$ LigN unfolds reversibly in response to chemical agents. We adapted a previously reported protocol $^{25}$ to obtain fully deadenylated ( $\mathrm{H} v$ dea-LigN) and fully adenylated ( $\mathrm{H} v$ ade-LigN) ligase and we explored the stability of the two enzyme isoforms in the presence of $\mathrm{KCl}$. Figure 1D shows the guanidinium chloride melting curves for $H v$ dea-LigN and $H v$ ade-LigN enzyme isoforms. Inspection of the melting curves clearly shows that unfolding does not obey a two-state model. This is not surprising given that $H v$ LigN is a multidomain protein of high molecular weigth. Previous thermodynamic characterization of the $\mathrm{NAD}^{+}$-dependent ligase $\mathrm{N}$ from Thermus scotoductus also showed a non-two-state behavior ${ }^{26}$. Instead, the experimental data fit well to a three-state model (fitting the experimental data to this model was validated according to the F-test analysis) and the solid lines in Figure 1D represent the best fits to this model. We employed the overall free energy upon unfolding $\left(\Delta G_{U-N}^{0}(0)=\Delta G_{U-I}^{0}(0)+\Delta G_{I-N}^{0}(0)\right)$ as a reporter for protein stability.

Free energies of $H v$ dea-LigN and $H v$ ade-LigN were determined at increasing concentrations of $\mathrm{KCl}$ (Figure $2 \mathrm{~A}$ ). In the absence of $\mathrm{K}^{+}$, $H v$ dea-LigN was more stable than $H v$ ade-LigN, providing an explanation for the lack of activity. This is in contrast to nonhalophilic ligases, where the protein becomes stabilised upon adenylation so the reaction can proceed. ${ }^{26} \mathrm{KCl}$, in turn, stabilised both isoforms, showing a linear dependence with the molar concentration of salt (Figure $2 \mathrm{~A}$ ), with slopes $\left(m_{K C l}\right)$ shown in Table S1. Interestingly, $m_{K C l}\left(H v\right.$ ade-LigN) was higher than $m_{K C l}(H v$ dea$\mathrm{LigN}$ ) and, at a concentration around $1 \mathrm{M}, \mathrm{H} v$ ade-LigN became more stable than the deadenylated form. The apparent populations of the adenylated and deadenylated species as a function of $\mathrm{KCl}$ concentration (obtained from the free energy values) were highly consistent with the experimental enzyme activity values (Figure 2B): at $\mathrm{KCl}$ concentrations below $1 \mathrm{M}$ there was no enzyme activity, in agreement with $H v$ dea-LigN as the only populated molecule. At concentrations higher than this value, $H v$ ade-LigN became thermodynamically more stable and the enzyme started showing ligase activity.

Ligase enzyme activity tuning in the presence of $\mathrm{Na}^{+} . \mathrm{NaCl}$ had a negligible effect on $H v$ LigN catalytic activity, even at very high a

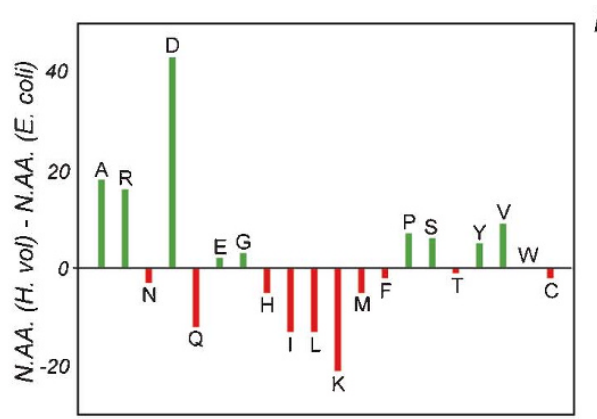

$c$

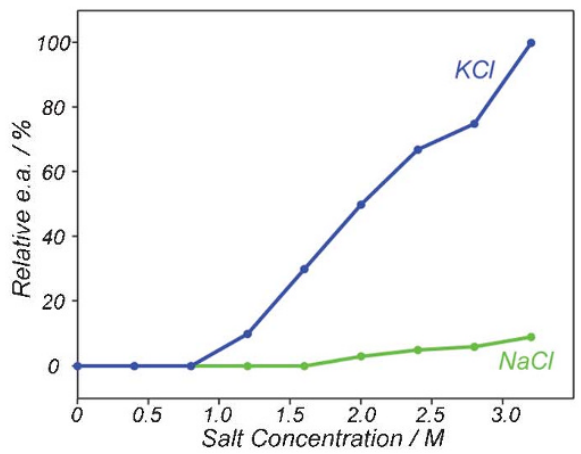

$b$
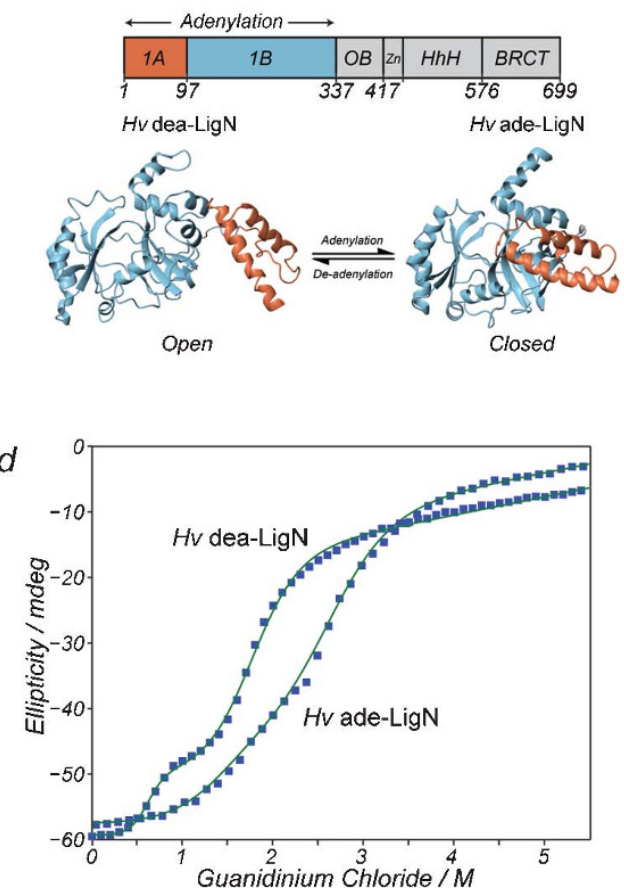

Figure $1 \mid$ (a) Amino acid composition difference between the primary sequences of $H$. volcanii and $E$. coli Lig N. Green (red) bars indicate an increase (decrease) of the given amino acid. (b) Top. Domain structure for the Hv LigN enzyme. The adenylation domain is divided into two subdomains: $1 \mathrm{~A}$ (orange) and 1B (light blue). Bottom. Modelled structures of the de-adenylated (open, left) and adenylated (closed, right) conformations. The model was obtained using the homology modelling program Phyre. ${ }^{21}$ (c) Relative enzyme activity as a function of the salt concentration and referenced to the catalytic activity in the presence of 3.2 M KCl. (d) Representative examples of the chemical denaturation profiles monitored by CD, for $H v$ dea-LigN and $H v$ ade-LigN. Solid lines correspond to the best fit using a three-state model. 
a

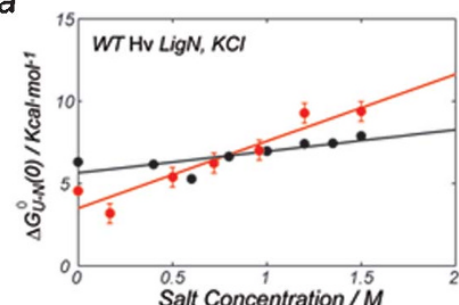

c

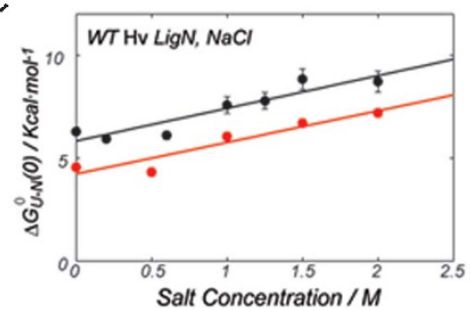

e

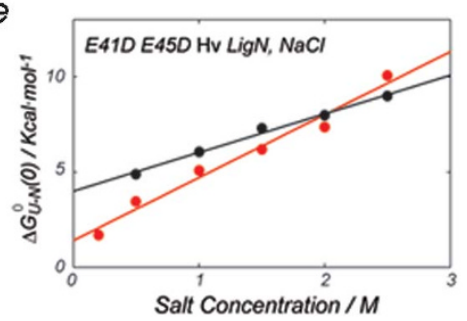

$b$

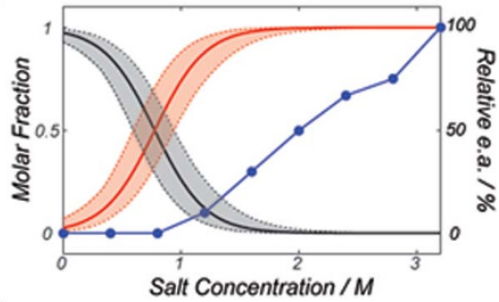

$d$

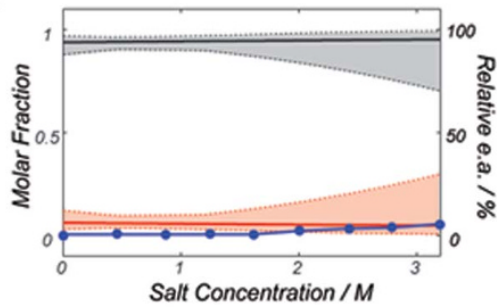

$f$

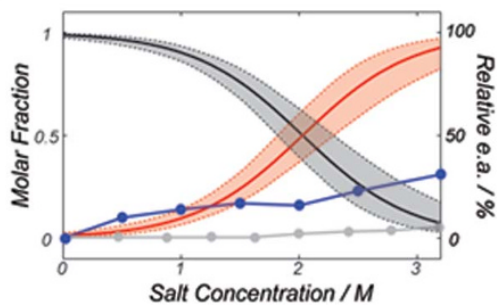

Figure $2 \mid$ Plots of the free energy upon unfolding $\left(\Delta \mathrm{G}_{\mathrm{U}-\mathrm{F}}\right)$ versus the molar concentration of salt are shown in panels (a), (c) and (e). The salt and the protein used are indicated in the panels. $H v$ dea-LigN and $H v$ ade-LigN are represented by black and red circles, respectively. A linear dependence between stability and the molar concentration of salt was found and the solid lines correspond to the linear regressions. The apparent populations of $H v$ dea-LigN and $H v$ ade-LigN are plotted in panels (b), (d) and (f) using the same colour code (left ordinate axes). The shaded areas in red (black) correspond to the estimated uncertainties for the $H v$ ade-LigN ( $H v$ dea-LigN) populations. The blue circles in the same panels represent the enzyme activity (normalised with respect to $3.2 \mathrm{M} \mathrm{KCl}$, right ordinate axes). In panel (f), WT $\mathrm{Hv}$ LigN activity as a function of $\mathrm{NaCl}$ concentration is also shown in gray.

concentrations of this salt. We reproduced the thermodynamic characterization of the adenylated and deadenylated forms of $\mathrm{Hv}$ LigN at increasing concentrations of $\mathrm{NaCl}$ (up to $2.0 \mathrm{M}$ ) and the results are shown in Figures $2 \mathrm{C}$ and $2 \mathrm{D}$. Interestingly, $\mathrm{Hv}$ deaLigN was more stable than $H v$ ade-LigN at all tested concentrations of $\mathrm{NaCl}$ (Figure 2C), and the equilibrium was never displaced towards the adenylated form. In other words, the red and black lines in Figures $2 \mathrm{C}-\mathrm{D}$ do not cross $\left(m_{\mathrm{NaCl}}(\mathrm{H} v\right.$ ade-LigN $) \approx$ $m_{\mathrm{NaCl}}(\mathrm{H} v$ dea-LigN)). To our knowledge, this is the first report for differential stabilisation of a protein induced by $\mathrm{KCl}$ when compared to $\mathrm{NaCl}$, even though computer simulations had predicted a minor stabilising effect for the $\mathrm{Na}^{+}$due to the higher affinity of this cation for the carbonyl groups of the protein. ${ }^{27-29}$

We have recently shown that a change in the hydrophobic content of the protein exposed to the solvent can modulate the salt effect on its stability. ${ }^{8}$ To test whether this strategy is useful in restoring enzyme activity, we created two very conservative mutations (E41D and E45D) in the 1A domain of $H v$ LigN that slightly alter the hydrophobic character of the protein by removing two methylene groups without affecting the overall charge. The circular dichroism spectrum for the mutant was identical to wild type $H v$ LigN (Figure S2), indicating that no structural perturbation occurred upon mutation. Enzyme activity for the mutant was tested as a function of $\mathrm{NaCl}$ and $\mathrm{KCl}$ concentration. In the presence of $\mathrm{NaCl}$, there was a clear recovery of catalytic activity upon mutation (Figures 3 and S1). A similar increase in the activity of the mutated enzyme was also observed when $\mathrm{KCl}$ was added instead, and the enzyme starts being catalytically active at a much lower concentration of this salt. Free energies of the $H v$ ade-LigN and $H v$ deaLigN isoforms for the engineered protein showed that the two species were stabilised by $\mathrm{NaCl}$ with different slopes (Figure $2 \mathrm{E}-\mathrm{F}$ ), progressively favouring the adenylated form, the key requisite to trigger the reaction. Thus, very subtle modifications in the protein's surface can modulate salt-induced enzyme activity, a property that could be exploited for the biotechnological use of these enzymes in algaculture.

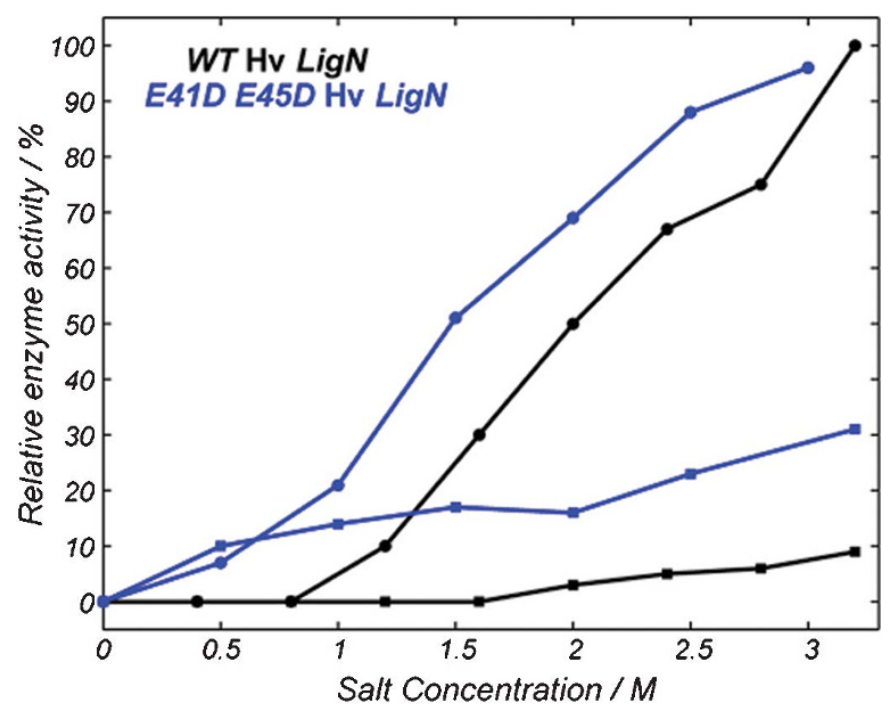

Figure 3 Dependence of the enzyme activity with the molar salt concentration (referenced to the wild type catalytic activity in the presence of 3.2 M KCl). Squares (circles) represent the enzyme activity of $\mathrm{Hv} \mathrm{LigN}$ in the presence of $\mathrm{NaCl}(\mathrm{KCl})$. Wild type and mutant proteins are shown in black and blue, respectively, as indicated in the figure. 
a

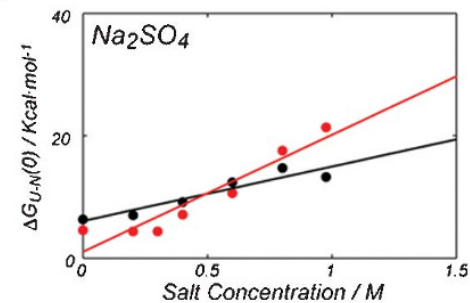

$c$

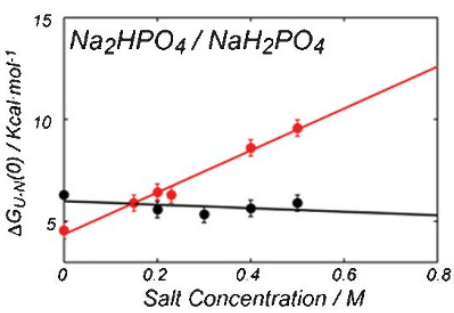

$b$

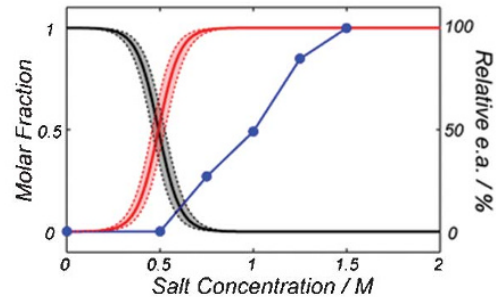

$d$

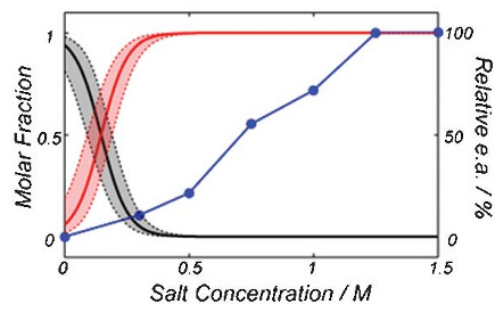

Figure $4 \mid$ Plots of the free energy upon unfolding $\left(\Delta G_{U-F}^{0}\right)$ for WT $H v$ LigN versus the molar concentration of salt are shown in panels (a) and (c) for the salts indicated in the panels. $H v$ dea-LigN and $H v$ ade-LigN are represented by black and red circles, respectively. A linear dependence between stability and the molar concentration of salt was found and the solid lines correspond to the linear regressions. The populations of $H v$ dea-LigN and $H v$ ade-LigN are plotted in panels (b) and (d) using the same colour code (left ordinate axes). The shaded areas in red (black) correspond to the estimated uncertainties for the $H v$ ade-LigN ( $H v$ dea-LigN) populations. The blue circles in the same panels represent the enzyme activity (normalised with respect to $3.2 \mathrm{M} \mathrm{KCl}$, right ordinate axes).

Effect of the anion on Hv LigN catalytic activity. To account for the effect of the anion, we also explored the stability of wild type $H v$ adeLigN and $H v$ dea-LigN in the presence of other salts (sodium sulphate and sodium phosphate). Sodium is chosen as counterion instead of potassium due to the higher solubility of guanidinium chloride in the presence of sodium salts. In both cases, the cosolute preferentially stabilised $H v$ ade-LigN and there was also a good agreement between the relative population of the two species and the catalytic activity of the enzyme (Figure 4). Consistent with data reported in other systems, ${ }^{30,31}$ protein stabilisation by salt is the sum of the contributions from the anion and the cation.

\section{Discussion}

Given the variety of possible effects that salt has on catalytic activity, its relationship with the adaptation of enzymes to hypersaline media is apparently complex. Here, we have investigated $H v \operatorname{LigN}$, a halophilic enzyme that requires $\mathrm{KCl}$ in order to be active. Prior to catalysis, Hv LigN undergoes cofactor binding (adenylation). The low stability of the adenylated form in the absence of salt (lower than the apo-enzyme) reveals that this procedure is not spontaneous, providing an explanation for the lack of activity of the enzyme under these conditions. The inactivation at low ionic strength may be an exclusive feature of this enzyme (perhaps shared by other halophilic enzymes as well). In support of this idea, a closely related $\mathrm{NAD}^{+}$dependent DNA ligase from Thermus scotoductus becomes more stable upon adenylation in the absence of salt ${ }^{26}$, as expected for a functional non-halophilic ligase.

Why does $\mathrm{KCl}$ preferentially stabilise the $H v$ ade-LigN species? The effect of salt on protein stability can be understood as the balance between two contributions: $:^{32}$ i) the non-specific (weak) interactions between the co-solute and the protein backbone that favour extended conformations and ii) the solvation of the hydrophobic moiety of the biomolecule that stabilises compact folding of the protein. According to our structural model, $H v$ ade-LigN buries $331 \AA^{2}$ of its surface to the solvent, adopting a more closed conformation than the deadenylated species. We and others have shown that a reduction in the area accessible to the solvent results in higher stabilisation induced by the salt. ${ }^{3-35}$ Thus, $\mathrm{KCl}$ preferentially stabilises the more compact $H v$ ade-LigN and this in turn results in the activation of the enzyme. On the other hand, upon $\mathrm{NaCl}$ addition, a compensation of both mechanisms occurs and the equilibrium cannot be displaced towards the adenylated form. This mechanism can also be extrapolated to other halophilic enzymes such as malate dehydrogenase $(\mathrm{Hm}$ $\mathrm{MalD})^{36}$ and lipase $\mathrm{C}(\mathrm{Hm} \text { LipC })^{37}$ from Haloarcula marismortui that also require $\mathrm{KCl}$ to be active. It has been reported that salt favours the formation of oligomers (tetramers in the case of $\mathrm{Hm}$ MalD and dimers for $\mathrm{Hm}$ LipC), the only forms for these enzymes that are catalytically active. ${ }^{37,38}$ This simple mechanism of stabilization paves the way for potential biotechnological applications. As an example, in Figure 3 we have shown how minimal protein surface modifications successfully modulate the stability dependence of a protein induced by the salt.

In summary, halophilic enzymes all share a very biased amino acid composition shaped by evolution and this signature appears to be a universal trait. ${ }^{2}$ Our results show that this primary sequence fundamentally alters the populations of the different states of the protein, favouring the folded versus the unfolded state, the closed versus the open conformation and the oligomeric versus the monomeric form. In cases where the enzyme reaction coordinate implies a conformational change, differential stabilisation of the species in the reaction pathway (as in $H v \operatorname{LigN}$ ) will also result in indirect modulation of the catalytic efficiency. The fact that this is an apparent effect is consistent with the very different modulations that $\mathrm{KCl}$ (or $\mathrm{NaCl}$ ) can exert on the activity of halophilic enzymes.

\section{Methods}

Equilibrium denaturation experiments. The effect of inorganic salts on the stability of $H v \operatorname{LigN}$ was determined by measuring equilibrium denaturation at increasing concentrations of the desired co-solute. The following salts were considered: sodium and potassium to explore the effect of cations, and sulfate, phosphate and chloride for the anion series. A JASCO J-810 spectropolarimeter with a quartz cuvette of $1 \mathrm{~cm}$ path length was used to monitor the denaturation experiments by means of circular dichroism. CD experiments were monitored at $222 \mathrm{~nm}$ with a bandwidth of $4 \mathrm{~nm}$ and collection of data every 0.2 degrees. Guanidinium chloride denaturation experiments employed an initial volume of $1.7 \mathrm{~mL}$ containing the protein $(0.6-1.3$ $\mu \mathrm{M})$ in $20 \mathrm{mM}$ phosphate buffer at $\mathrm{pH} 8.0$ and at the appropriate concentration of the inorganic salt. Protein denaturation was achieved by the addition of aliquots (performed with an automatic titrator) of a solution with the same protein concentration and buffer conditions but also containing a solution of denaturant agent. The concentration of the denaturant agent was adjusted by measuring the refractive index. The range of co-solute concentrations tested was limited by the reduced solubility of the inorganic salts in the presence of denaturant. The denaturant concentration was lowered to expand this solubility without compromising the 
proper determination for the unfolded state baseline (see below), with final denaturant concentrations falling in the ranges between 4.8 M-6.9 M. Between two and four independent measurements were obtained for each experimental condition and duplicate points were used to obtain an estimation of the error.

Data analysis was completed with in-house built scripts programmed in Matlab (Simulink). Guanidinium chloride denaturation data monitored by CD were processed assuming the linear extrapolation method ${ }^{39}$ adapted to three states. Specifically, the equation used for the raw data fitting was: ${ }^{40}$

$$
\theta_{o b s}=\frac{\theta_{N}+\left\{\theta_{I}+\left(\theta_{U}+q[\text { denat }] \cdot e^{b}\right) \cdot e^{a}\right\}}{1+\left(1+e^{b}\right) \cdot e^{a}}
$$

where

$$
a=\frac{-\Delta G_{I-N}^{0}(0)+m_{I N} \cdot[\text { denat }]}{R T} \text { and } b=\frac{-\Delta G_{U-I}^{0}(0)+m_{U I} \cdot[\text { denat }]}{R T}
$$

and where $\Delta G_{I-N}^{0}(0)$ and $\Delta G_{U-I}^{0}(0)$ are the free energy differences between the intermediate (I) and the native state $(\mathrm{N})$ and between I and the unfolded state (U) respectively in the absence of denaturant. $\theta_{o b s}, \theta_{N}, \theta_{I}$ and $\theta_{U}$ are the ellipticities observed, for the native state, for the intermediate and for the unfolded state respectively. $m_{I N}$ and $m_{N U}$ are the slopes of the transitions and $\mathrm{q}$ is an empirical factor that accounts for the linear dependence of the CD signal in the unfolded state. The total free energy of unfolding in the absence of denaturant corresponds to:

$$
\Delta G_{U-N}^{0}(0)=\Delta G_{U-I}^{0}(0)+\Delta G_{I-N}^{0}(0)
$$

The uncertainties in the free energies were propagated by Montecarlo analysis (with 15000 steps) to provide an estimation of the error in the populations as a function of the concentration (Figures 2B, 2D, 2F, S2B, S2D).

DNA ligase activity assay. DNA ligase assays were performed as previously described by Poidevin and MacNeill ${ }^{19}$ with minor modifications. Ligase reactions $(20 \mu \mathrm{L})$ contained $1.25 \mu \mathrm{g}$ of $\mathrm{Hv} \operatorname{LigN}$ in $20 \mathrm{mM}$ Tris- $\mathrm{HCl}\left(\mathrm{pH} 7.6\right.$ at $25^{\circ} \mathrm{C}$ ), $3.2 \mathrm{M} \mathrm{KCl}$ (unless otherwise indicated), $25 \mathrm{mM}$ potassium acetate, $10 \mathrm{mM}$ magnesium acetate, $10 \mathrm{mM}$ DTT, $1 \mathrm{mM} \mathrm{NAD}^{+}, 0.01 \%$ Triton $\times 100,3 \mu \mathrm{L}$ of $500 \mu \mathrm{g} / \mathrm{mL}$ of BstEIIdigested $\lambda$ DNA (New England Biolabs) and the test concentration of co-solute. Reactions were run at $45^{\circ} \mathrm{C}$ for 10 minutes and stopped by the addition of $4 \mu \mathrm{L}$ of $50 \mathrm{mM}$ EDTA. The samples were then desalted using the MinElute reaction clean-up kit (Qiagen), according to the manufacturer's instructions, eluted in $20 \mu \mathrm{L}$ of EB buffer ( $10 \mathrm{mM}$ Tris $\mathrm{pH} 8.0$ ), heated to $65^{\circ} \mathrm{C}$ for 5 minutes and chilled on ice for 5 minutes prior to loading onto a $1 \%$ agarose gel prepared in TAE buffer. The gel contained $1 \times$ Sybr $_{\circledast}$ Safe DNA gel stain (Invitrogen) and was run at $120 \mathrm{~V}$ for 60 minutes. Gels were visualized in the Chemidoc ${ }^{\mathrm{TM}}$ XRS System (BioRad) and band intensities were determined using ImageJ 1.410 analysis software (Wayne Rasband, National Institutes of Health, USA). Activity was calculated by measuring the net intensity of the product band (fragment I+IV) relative to the non-substrate fragments (bands II + III, V + VI or VII). The assay was tested in the presence of sodium and potassium, sulfate, phosphate, and chloride. In the case of the phosphate anion, the buffer was incubated at $75^{\circ} \mathrm{C}$ for $10 \mathrm{~min}$ prior to running the ligase activity test, due to the low solubility of the magnesium phosphate.

Adenylation / de-adenylation assay. Due to the lower stability of the halophilic ligase, the adenylation and de-adenylation reactions were adapted from the original protocol. ${ }^{25}$ For de-adenylation, an 80 -fold excess of nicotinamide mononucleotide (NMN) and magnesium chloride was added to the protein (concentration of approximately $30 \mu \mathrm{M}$ ). The mixture was then incubated for between 75 and $100 \mathrm{~h}$ at $25{ }^{\circ} \mathrm{C}$ prior to purification by gel filtration chromatography (Superdex 75,

Amersham). Adenylation was achieved by adding an 80 -fold excess of $\mathrm{NAD}^{+}$to the protein and subsequent incubation of the mixture for $20 \mathrm{~h}$ at the same temperature.

1. Lanyi, J.K. Salt-dependent properties of proteins from extremely halophilic bacteria. Bacteriol Rev 38, 272-90 (1974).

2. Paul, S., Bag, S.K., Das, S., Harvill, E.T. \& Dutta, C. Molecular signature of hypersaline adaptation: insights from genome and proteome composition of halophilic prokaryotes. Genome Biol 9, R70 (2008).

3. Fukuchi, S., Yoshimune, K., Wakayama, M., Moriguchi, M. \& Nishikawa, K. Unique amino acid composition of proteins in halophilic bacteria. J Mol Biol 327, 347-57 (2003)

4. Esclapez, J. et al. Analysis of acidic surface of Haloferax mediterranei glucose dehydrogenase by site-directed mutagenesis. FEBS Lett 581, 837-42 (2007).

5. Kastritis, P.L., Papandreou, N.C. \& Hamodrakas, S.J. Haloadaptation: insights from comparative modeling studies of halophilic archaeal DHFRs. Int J Biol Macromol 41, 447-53 (2007).

6. Polosina, Y.Y., Zamyatkin, D.F., Kostyukova, A.S., Filimonov, V.V. \& Fedorov O.V. Stability of Natrialba magadii NDP kinase: comparisons with other halophilic proteins. Extremophiles 6, 135-42 (2002).

7. Ebel, C., Faou, P., Kernel, B. \& Zaccai, G. Relative role of anions and cations in the stabilization of halophilic malate dehydrogenase. Biochemistry 38, 9039-47 (1999)

8. Tadeo, X. et al. Structural basis for the aminoacid composition of proteins from halophilic archea. PLoS Biol 7, e1000257 (2009).
9. Wright, D.B., Banks, D.D., Lohman, J.R., Hilsenbeck, J.L. \& Gloss, L.M. The effect of salts on the activity and stability of Escherichia coli and Haloferax volcanii dihydrofolate reductases. J Mol Biol 323, 327-44 (2002).

10. Madern, D., Ebel, C. \& Zaccai, G. Halophilic adaptation of enzymes. Extremophiles 4, 91-8 (2000).

11. Mevarech, M., Frolow, F. \& Gloss, L.M. Halophilic enzymes: proteins with a grain of salt. Biophys Chem 86, 155-64 (2000).

12. Nagashima, K., Mitsuhashi, S., Kamino, K. \& Maruyama, T. Cyclosporin A sensitive peptidyl-prolyl cis-trans isomerase in a halophilic archaeum, Halobacterium cutirubrum. Biochem Biophys Res Commun 198, 466-72 (1994).

13. Jolley, K.A. et al. Dihydrolipoamide dehydrogenase from the halophilic archaeon Haloferax volcanii: homologous overexpression of the cloned gene. J Bacteriol 178, 3044-8 (1996).

14. Holmes, M.L. et al. Purification and analysis of an extremely halophilic betagalactosidase from Haloferax alicantei. Biochim Biophys Acta 1337, 276-86 (1997).

15. Brown-Peterson, N.J. \& Salin, M.L. Purification of a catalase-peroxidase from Halobacterium halobium: characterization of some unique properties of the halophilic enzyme. J Bacteriol 175, 4197-202 (1993).

16. Ebel, C. et al. Solvent interactions of halophilic malate dehydrogenase. Biochemistry 41, 13234-44 (2002).

17. Zhao, A., Gray, F.C. \& MacNeill, S.A. ATP- and NAD+-dependent DNA ligases share an essential function in the halophilic archaeon Haloferax volcanii. Mol Microbiol 59, 743-52 (2006).

18. Gajiwala, K.S. \& Pinko, C. Structural rearrangement accompanying NAD + synthesis within a bacterial DNA ligase crystal. Structure 12, 1449-59 (2004).

19. Poidevin, L. \& MacNeill, S.A. Biochemical characterisation of LigN, an NAD+ dependent DNA ligase from the halophilic euryarchaeon Haloferax volcanii that displays maximal in vitro activity at high salt concentrations. BMC Mol Biol 7, 44 (2006).

20. Tomkinson, A.E., Vijayakumar, S., Pascal, J.M. \& Ellenberger, T. DNA ligases: structure, reaction mechanism, and function. Chem Rev 106, 687-99 (2006).

21. Bennett-Lovsey, R.M., Herbert, A.D., Sternberg, M.J. \& Kelley, L.A. Exploring the extremes of sequence/structure space with ensemble fold recognition in the program Phyre. Proteins 70, 611-625 (2007).

22. Arnold, K., Bordoli, L., Kopp, J. \& Schwede, T. The SWISS-MODEL workspace: a web-based environment for protein structure homology modelling. Bioinformatics 22, 195-201 (2006).

23. Bieger, B., Essen, L.O. \& Oesterhelt, D. Crystal structure of halophilic dodecin: a novel, dodecameric flavin binding protein from Halobacterium salinarum. Structure 11, 375-85 (2003).

24. Premkumar, L. et al. Three-dimensional structure of a halotolerant algal carbonic anhydrase predicts halotolerance of a mammalian homolog. Proc Natl Acad Sci U S A 102, 7493-8 (2005).

25. Timson, D.J. \& Wigley, D.B. Functional domains of an NAD+-dependent DNA ligase. J Mol Biol 285, 73-83 (1999).

26. Georlette, D. et al. Adenylation-dependent conformation and unfolding pathways of the NAD+-dependent DNA ligase from the thermophile Thermus scotoductus. Biophys J 86, 1089-104 (2004).

27. Hess, B. \& van der Vegt, N.F. Cation specific binding with protein surface charges. Proc Natl Acad Sci U S A 106, 13296-300 (2009).

28. Vrbka, L., Vondrasek, J., Jagoda-Cwiklik, B., Vacha, R. \& Jungwirth, P. Quantification and rationalization of the higher affinity of sodium over potassium to protein surfaces. Proc Natl Acad Sci U S A 103, 15440-4 (2006).

29. Dzubiella, J. Salt-specific stability of short and charged alanine-based alphahelices. J Phys Chem B 113, 16689-94 (2009).

30. Fayos, R., Pons, M. \& Millet, O. On the origin of the thermostabilization of proteins induced by sodium phosphate. J Am Chem Soc 127, 9690-1 (2005).

31. Gloss, L.M. \& Placek, B.J. The effect of salts on the stability of the H2A-H2B histone dimer. Biochemistry 41, 14951-9 (2002).

32. Baldwin, R.L. How Hofmeister ion interactions affect protein stability. Biophys J 71, 2056-63 (1996)

33. Pegram, L.M. \& Record, M.T., Jr. Hofmeister salt effects on surface tension arise from partitioning of anions and cations between bulk water and the air-water interface. J Phys Chem B 111, 5411-7 (2007).

34. Schellman, J.A. Protein stability in mixed solvents: a balance of contact interaction and excluded volume. Biophys J 85, 108-25 (2003).

35. Tadeo, X., Lopez-Mendez, B., Castano, D., Trigueros, T. \& Millet, O. Protein stabilization and the hofmeister effect: the role of hydrophobic solvation. Biophys $J$ 97, 2595-603 (2009).

36. Eisenberg, H. Life in unusual environments: progress in understanding the structure and function of enzymes from extreme halophilic bacteria. Arch Biochem Biophys 318, 1-5 (1995).

37. Rao, L. et al. Solution behavior and activity of a halophilic esterase under high salt concentration. PLoS One 4, e6980 (2009).

38. Dym, O., Mevarech, M. \& Sussman, J.L. Structural Features That Stabilize Halophilic Malate Dehydrogenase from an Archaebacterium. Science 267, 13441346 (1995). 
39. Santoro, M.M. \& Bolen, D.W. Unfolding free energy changes determined by the linear extrapolation method. 1. Unfolding of phenylmethanesulfonyl alpha-chymotrypsin using different denaturants.Biochemistry 27, 8063-8 (1988)

40. Vanhove, M. et al. Kinetic and thermodynamic consequences of the removal of the Cys-77-Cys-123 disulphide bond for the folding of TEM-1 beta-lactamase. Biochem J 321 ( Pt 2), 413-7 (1997).

\section{Acknowledgements}

The plasmid containing the $H$. volcanii ligase $\mathrm{N}$ was kindly provided by Stuart McNeill (University of Copenhagen). We are very grateful to Prof. Robert Baldwin (Standford University) for valuable comments on the manuscript. Support was provided from The Department of Industry, Tourism and Trade of the Government of the Autonomous Community of the Basque Country, from the Innovation Technology Department of the Bizkaia County, from the Ministerio de Ciencia y Tecnología (CTQ2009-10353/BQU and CSD2008-00005). G.O. acknowledges a fellowship from the Fundació "Obra Social” La Caixa.

\section{Author contributions}

G.O., A.L., X.T. and B.L. purified the proteins and performed the enzymatic assays. G.O. and X.T. measured the enzyme free energies in the presence of salts. O. M. calculated the protein models. G. O. and A.L. analyzed the data. G.O. and D.C. performed the mutagenesis studies. O.M. and G.O. designed the experiments and wrote the paper.

\section{Additional information}

Supplementary Information accompanies this paper at http://www.nature.com/ scientificreports

Competing financial interests: The authors declare no competing financial interests.

License: This work is licensed under a Creative Commons

Attribution-NonCommercial-ShareAlike 3.0 Unported License. To view a copy of this license, visit http://creativecommons.org/licenses/by-nc-sa/3.0/

How to cite this article: Ortega, G. et al. Halophilic enzyme activation induced by salts. Sci. Rep. 1, 6; DOI:10.1038/srep00006 (2011) 